\title{
Melanin production by Pseudomonas sp. and in silico comparative analysis of tyrosinase gene sequences
}

\author{
Seelam Siva Deepthi ${ }^{1}$, Mohan Reddy K. ${ }^{2}$, Neelam Mishra ${ }^{1}$, Dayanand AgSaR $^{1}$ * \\ ${ }^{1}$ Department of Microbiology, Gulbarga University, Kalaburagi, Karnataka, India \\ ${ }^{2}$ Department of Biotechnology, Gulbarga University, Kalaburagi, Karnataka, India
}

\begin{abstract}
Background. Melanin finds enormous applications in different industries for its unique photoprotective and anti-oxidant properties. Due to its emerging demand, scientific researchers are putting efforts to unravel more microorganisms with a potential of producing melanin on large scale. Hence, the present study was aimed at the isolation of extracellular melanin producing microorganisms from lime quarries of Karnataka, India. Besides this, the tyrosinase gene governing melanin synthesis in different organisms were compared in silico to understand its evolutionary aspects. Material and methods. Melanin producing microorganisms were screened on tyrosine gelatin beef extract agar medium. Potential isolate was explored for submerged production of melanin in broth containing L-tyrosine. Melanin was characterized by UV-Vis spectroscopy, thin layer and high performance liquid chromatographic techniques. Antibacterial activity of melanin was performed by agar well assay. Comparative tyrosinase gene sequence analysis was performed by using Geneious 2021.1 trial version software. Results. Pseudomonas otitidis DDB2 was found to be potential for melanin production. No antibacterial activity was exerted by the melanin against tested pathogens. The in silico studies showed that the common central domain of tyrosinase protein sequence of selected Pseudomonas sps. exhibited 100\% identity with the common central domain of Homo sapiens tyrosinase (NP_000363.1). Conclusions. Our study shows the production of melanin in good quantities by the isolate Pseudomonas otitidis DDB2 which can be explored for scale-up process. Since the melanin formed is of eumelanin type and the tyrosinase gene sequence of several Pseudomonas sp. showed relatedness to humans, this molecule may be further developed for sunscreen formulations.
\end{abstract}

Key words: Pseudomonas sps., Homo sapiens, natural pigment, melanogenic genes, common central domain

\section{Introduction}

Microorganisms serve as natural resources of bioactive molecules. Among various bioactive entities, microbial pigments have recently gained wide research interest because of their natural origin and safe application. Melanins are one such group of pigments with complex structures and physicochemical properties $(\mathrm{Ba}-$ nerjee et al., 2014). Most of the living organisms on the Earth produce melanin to protect themselves from detrimental effects of solar radiation (D'Ischia $\mathrm{M}$ et al., 2015). Although many microorganisms can produce melanin, their scaling up and extraction process require further optimization as melanin is produced inside melanosomes (Toledo et al., 2017). Some microorganisms such as Streptomyces, Nocardiopsis, Pseudomonas, $A^{-}$ pergillus, Auricularia, and Armillaria have attracted the attention of scientists because of their ability to produce extracellular melanin which can be easily extracted and purified (Kiran et al., 2014; Raman et al., 2015; Sun et al., 2016; El Naggar and El-Ewasy, 2017; Kiran et al., 2017; Ribera et al., 2019). Jalmi et al. (2012) reported maximum melanin production $(6.6 \mathrm{~g} / \mathrm{l})$ by the fungus Gliocephalotrichum simplex after 6 days of incubation. On the other hand, Streptomyces kathirae SC-1 produced $13.7 \mathrm{~g} / 1$ melanin after 5 days of incubation (Guo et al., 2014). Most of these microorganisms depend on the exogenous supply of precursors such as mono-/diphenols for extracellular melanin synthesis (Tran-Ly

\footnotetext{
* Corresponding author: Department of Microbiology, Gulbarga University, Kalaburagi-585106, Karnataka, India; e-mail: dayanandagsar@gmail.com
} 
et al., 2020). Melanin has gained importance because of its potential application in antioxidant and sunscreen products, organic semiconductor devices, biomedical devices, metal chelators, and dyeing agents (Ali Amal et al., 2011; Battistella et al., 2020; Tran-Ly et al., 2020). A study revealed that the thermal stability of the synthetic polymer polymethyl methacrylate was enhanced when eumelanin was added to the mixture. It was shown that melanin remarkably increased the decomposition temperature of the polymer by altering its radical initiated chain scission behavior (Shanmuganathan et al., 2011). A recent report showed the application of melanin as a colorant for soft contact lenses (Ahn et al., 2019). Another study described the use of melanin as a contrast agent in imaging application (Williams, 1994). Melanin extracted from Streptomyces sp. and Pseudomonas balearica shows significant antimicrobial activity against pathogenic bacteria such as Escherichia coli, Staphylococcus aureus, Lactobacillus vulgaris, Vibrio cholera, Proteus miralis, Salmonella typhae, Salmonella paratyphae, and Klebsiella oxytoca (Vasanthabharathi et al., 2011; Zerrad et al., 2014). Because of its unique properties such as heterogeneity, hydrophobicity, and negative charge, the structural analysis of natural melanin is challenging (Pralea et al., 2019).

Many microorganisms can synthesize melanin through multiple pathways. For instance, microorganisms such as Streptomyces glaucescens, Neurospora crassa, and $\mathrm{Ma}$ rinomonas mediterranea can produce melanin by using a pathway similar to that of mammals, which involves the enzyme tyrosinase (Serrano et al., 2004; Kamal et al., 2014; Toledo et al., 2017), while other microorganisms such as Aspergillus fumigatus, Talaromyces marneffei, and Sporothrix schenckii use the DHN pathway/malonyl coenzyme for melanin synthesis (Langfelder et al., 1998; Romero-Martinez et al., 2000; Woo et al., 2010; Cordero and Casadevall, 2017). Certain microorganisms such as Cryptococcus neoformans and Bacillus weihenstephanensisfollow the laccase enzyme pathway, while bacteria such as Vibrio cholerae, Hyphomonas sp., and Shewanella colwelliana use the homogentisic acid pathway for melanin synthesis (Kotob et al., 1995; Eisenman et al., 2007; Wang et al., 2011; Drewnowska et al., 2015). Melanins produced through the DHN pathway are localized intracellularly; consequently, they are difficult to purify and hence not desirable for large-scale production (Toledo et al., 2017). Therefore, microorganisms that pro- duce extracellular melanin within a short incubation period are of industrial interest and are relatively economical. An analysis of the melanogenic genes of different taxa would provide better understanding on the importance of their evolutionary aspects (Esposito et al., 2012). In view of this, the present study aimed to screen melanin-producing potential organisms isolated from harsh habitats such as lime quarries in Karnataka, India. The screening process was followed by characterization of melanin and assessment of its antibacterial property. In addition, an attempt was made for comparative analysis of tyrosinase gene, protein, and common central domain (CCD) sequences of selected organisms.

\section{Materials and methods}

\section{Screening of soil samples for melanin-producing microorganisms}

Eleven soil samples collected from lime quarries in Karnataka, India, were pretreated and initially isolated on starch casein agar medium. The isolates for extracellular production of tyrosinase and melanin were screened by the streak plate culture method on a tyrosine gelatin beef extract (TGB) agar medium containing tyrosine $0.5 \%$, gelatin $0.5 \%$, beef extract $0.3 \%$, agar $2 \%$, and distilled water with $\mathrm{pH}$ 8.0. The inoculated plates were incubated at $40^{\circ} \mathrm{C}$ for 5 days. On the fifth day of incubation, the plates were visually assessed for zones of catalysis, and different intensities of brown color were recorded as positive for the synthesis of extracellular tyrosinase and melanin by the test isolates (Shivaveerakumar et al., 2013).

\section{Identification of the potential isolate}

The potential isolate for melanin production was identified preliminarily by Gram staining (Bartholomew and Mittwer, 1952) and motility test by the hanging drop method on a cavity slide. Molecular characterization of the isolate was performed by $16 \mathrm{~S}$ rRNA gene sequencing using the primers $27 \mathrm{~F}$ ( $5^{\prime}$-AGAGTTTGATCMTGGCT CAG $\left.-3^{\prime}\right)$ and $1525 \mathrm{R}\left(5^{\prime}-\mathrm{AAGGAGGTGATCCAGCC}-3^{\prime}\right)$ (Thorat and Dastager, 2018). The ability of the bacterial isolate to utilize different simple carbohydrates $(0.3 \%$ $\mathrm{w} / \mathrm{v}$ ) such as glucose, fructose, sucrose, maltose, raffinose, and xylose as the sole source of carbon was assessed in $\mathrm{M} 9$ minimal medium at $37^{\circ} \mathrm{C}$ for $24 \mathrm{~h}$ (Schleheck et al., 2009). The growth of bacteria was determined by measuring the optical density (OD) at $600 \mathrm{~nm}$. 


\section{Optimization of physicochemical variables on melanin production}

The influence of various factors on melanin production by a potential isolate was studied in L-tyrosine broth incubated at $40^{\circ} \mathrm{C}$ for $24 \mathrm{~h}$ with agitation speed of $120 \mathrm{rpm}$ (Madhusudhan et al., 2014). The effects of $\mathrm{pH}$ (6.0-10.0), temperature $\left(30-50^{\circ} \mathrm{C}\right)$, and L-tyrosine concentration $(0-2 \mathrm{~g} / \mathrm{l})$ were studied. The effects of different nitrogen sources $(0.5 \%$ each of beef extract, peptone, gelatin, yeast extract, and casein), metal salts (0.01\% each of $\mathrm{CuCl}_{2}, \mathrm{CoCl}_{2}, \mathrm{CuSO}_{4}, \mathrm{MnCl}_{2}$, and $\mathrm{MgCl}_{2}$ ), and salinity $(0-2 \% \mathrm{NaCl})$ on the production of melanin by the potential isolate were also assessed. All the experiments were performed in triplicate, and the results are presented as mean \pm standard deviation.

\section{Production and purification of melanin}

Submerged production of extracellular melanin by the potential isolate was accomplished in an optimized broth with agitation speed of $120 \mathrm{rpm}$ for $24 \mathrm{~h}$. The production broth was centrifuged after $24 \mathrm{~h}$ of incubation to separate the cell mass from other debris. The supernatant obtained was acidified with $1 \mathrm{~N} \mathrm{HCl}$ till it reached $\mathrm{pH} 2.0$ and was allowed to stand for $96 \mathrm{~h}$ for melanin aggregation. The aggregate formed was separated and purified by centrifugation at $10000 \mathrm{rpm}$ for $20 \mathrm{~min}$, followed by washing with ether, ethanol, acetone, and deionized water to remove impurities (Fava et al., 1993). The purified melanin was air-dried and stored at $4{ }^{\circ} \mathrm{C}$ for further use.

\section{Characterization of melanin}

Purified melanin was assessed for its solubility profile in different solvents such as water $(\mathrm{pH} 7.0), 1 \mathrm{~N} \mathrm{HCl}$, alkaline solutions $\left(1 \mathrm{~N} \mathrm{NaOH}\right.$ and $\left.1 \mathrm{~N} \mathrm{NH}_{4} \mathrm{OH}\right)$ and organic solvents such as dimethyl sulfoxide (DMSO), dimethyl formamide (DMF), methanol, ethanol, n-butanol, ethyl acetate, acetone, n-hexane, and diethyl ether. Other physical tests such as precipitation of melanin with $1 \% \mathrm{FeCl}_{3}$ and bleaching with alkaline $\mathrm{H}_{2} \mathrm{O}_{2}(10 \%)$ were performed as reported previously (Gibson and George, 1998). Melanin was characterized by spectroscopy and chromatographic methods. In brief, melanin was dissolved in $0.1 \mathrm{~N}$ $\mathrm{NaOH}$ and scanned in the range of $200-800 \mathrm{~nm}$ UV-Vis spectroscopy. Thin layer chromatography (TLC) was performed by using ethyl acetate and n-hexane $(7: 3)$ along with $0.1 \%$ formic acid as the mobile phase. Highperformance liquid chromatography (HPLC) was per- formed by dissolving melanin $(10 \mu \mathrm{g})$ in $10 \mu \mathrm{l}$ of diluted $\mathrm{KOH}(0.01 \mathrm{~N})$ solution, and the volume was made up to $1 \mathrm{ml}$ with methanol and filtered through $0.2 \mu \mathrm{m}$ Millex membrane (MF-Millipore ${ }^{\mathrm{TM}}$ ). The mobile phase comprised $0.1 \%$ formic acid and methanol in the ratio of $85: 15$, and the detection was performed with a C18 Kinetex ${ }^{\mathrm{TM}}$ $5 \mu \mathrm{m}, 260 \times 4.6 \mathrm{~mm}$ column at the flow rate of $1 \mathrm{ml} / \mathrm{min}$ at $222 \mathrm{~nm}$ in the diode array detector (Singh et al., 2018).

\section{Antibacterial activity of melanin}

The agar well diffusion assay was performed to assess the antibacterial activity of the melanin extracted from the bacterial isolate against the test pathogenic bacterial species E. coli, $P$. aeruginosa, Streptococcus entericus, and $B$. subtilis (Arun et al., 2015). The culture medium of each test isolate incubated for $18 \mathrm{~h}$ in nutrient broth was swabbed separately on the surface of sterile nutrient agar plates. Eight wells of $10 \mathrm{~mm}$ diameter were punched on each plate and loaded with $100 \mu \mathrm{l}$ of different concentrations of melanin (300, 250, $200,150,100$, and $50 \mu \mathrm{g} /$ well and labeled as A-F, respectively), streptomycin ( $1 \mathrm{mg} / \mathrm{ml})$, and DMSO. All the plates were incubated at $35^{\circ} \mathrm{C}$ for $24 \mathrm{~h}$. Streptomycin was used as a positive control, while the solvent DMSO was added as a negative control. The diameter of the zones of inhibition were measured to assess the antibacterial activity.

\section{Comparative analysis of tyrosinase gene and protein sequences from selected organisms}

The sequences of the tyrosinase gene from selected organisms were compared and analyzed to find the closest match with the human tyrosinase gene. A total of 72 tyrosinase genes and their reference protein sequences from prokaryotic and eukaryotic organisms (Table 1) were retrieved from the National Center for Biotechnology Information (NCBI) in FASTA format. The reference protein sequences were analyzed to find conserved motifs by using the KEGG's Motif Search web tool allied with Pfam database. The selected gene sequences, their respective proteins, and conserved motif sequences were separately subjected to multiple alignment using Clustal Omega 1.2.2 in Geneious software 10.2. After the multiple alignment, a distance matrix of sequences and phylogenetic tree were constructed to comprehend the evolutionary relationship between the tyrosinase sequences of selected organisms and Homo 
Table 1. List of selected organisms for the retrieval of tyrosinase gene and protein sequences

\begin{tabular}{|c|c|c|c|}
\hline Pseudomonas & Streptomyces & Fungi & Others \\
\hline P. putida NBRC 14164 & S. rimosus ATCC 10970 & Agaricus bisporus & Homo sapiens (TYRP1 gene) \\
\hline P. helleri DSM 29165 & S. griseus griseus NBRC 13350 & Aspergillus niger strain $\mathrm{PA} 2$ & Sepia officinalis \\
\hline P. frederiksbergensis FW305 & S. californicus NRRL B-2988 & Colletotrichum karsti & Vibrio nigripulchritudo \\
\hline$P$ poae LMG 21465417 & S. clavuligerus F1D7 (plasmid) & Lasiodiplodia theobromae & \\
\hline P. lactis SS101 & S. scabei 87.22 & Geosmithia morbida & \\
\hline P. chlororaphis qlu-1 & S. venezulae ATCC 21113 & Arthroderma uncinatum & \\
\hline P. simiae PCL1751 & S. zinciresistens $\mathrm{K} 42$ & Daldinia childiae & \\
\hline$P$. yamanorum LBUM636 & S. roseochromogenus subsp. oscitans DS 12.976 & Dothidotthia symphoricarpi CBS 119687 & \\
\hline P. veronii & S. pluripotens MUSC 135 & Lindgomyces ingoldianus & \\
\hline P. synxantha R6-28-08 & S. olivochromogenes DSM 40451 & Macroventuria anomochaeta & \\
\hline P. proteolytica LMG 22710 & S. virginiae NRRL ISP-5094 & Pseudovirgaria hyperparasitica & \\
\hline P. gingeri A6001 & S. subrutilus ATCC 27467 & Aaosphaeria arxii CBS 175.79 & \\
\hline P. moraviensis TYU6 & S. canus DSM 40017 & Didymella exigua CBS 183.55 & \\
\hline$P$. asiatica RYU5 & S. avermitilis MA-4680 & Zymoseptoria tritici IPO323 & \\
\hline P. orientalis $8 \mathrm{~B}$ & S. flavovariabilis NRRL B-16367 & Grifola frondosa & \\
\hline P. monteilii SB3101 & S. azureus ATCC 14921 & Talaromyces marneffei ATCC 18224 & \\
\hline P. azotoformans & S. atroolivaceous NRRL ISP-5137 & Blastomyces gilchristii & \\
\hline P. fluorescens $\mathrm{Pf} 0-1$ & S. xanthophaeus NRRL B-5414 & Pyricularia oryzae $70-15$ & \\
\hline P. synxantha NCTC 10696 & S. hygroscopicus subsp. jinggangensis TL01 & Microsporum canis CBS 113480 & \\
\hline \multirow[t]{9}{*}{ P. proteolytica WS 5126} & S. cyaneus CGMCC 4.1671 & Sodiomyces alkalinus $\mathrm{F} 11$ & \\
\hline & S. violaceoruber $\mathrm{S} 21$ & & \\
\hline & S. katrae S3 & & \\
\hline & S. fodineus TW1S1 & & \\
\hline & S. glaucescens GLA.O & & \\
\hline & S. fungicidicus TXX3120 & & \\
\hline & S. lavendulae subsp. lavendulae CCM 3239 & & \\
\hline & S. rubrolavendulae MJM4426 & & \\
\hline & S. wuyuanensis CGMCC 4.7042 & & \\
\hline
\end{tabular}




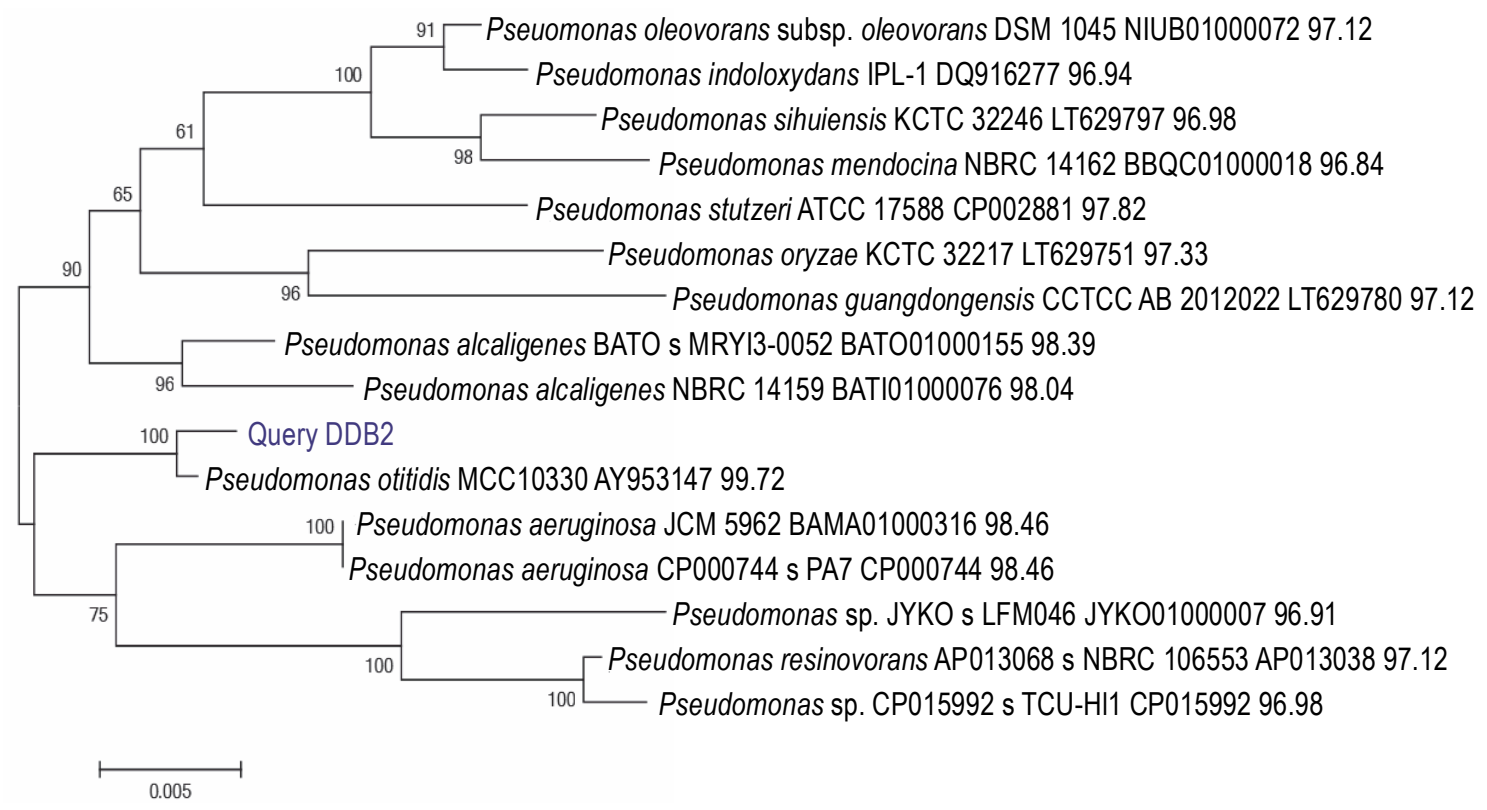

Fig. 1. Phylogenetic tree of Pseudomonas otitidis DDB2

sapiens (NM_000372.5) (Esposito et al., 2012). H. sapiens was selected as the out group while constructing the phylogenetic tree.

\section{Results}

A total of 58 isolates were obtained from lime quarry soil samples collected from Karnataka, India. All the 58 isolates were screened for melanin production, and only six isolates produced dark brown pigment on the TGB agar medium. Among the six positive isolates, the bacterial isolate named DDB2 (D: Name of Research Guide, Prof. Dayanand; D: Name of the Research Student, Deepthi; B: Type of isolate, bacteria; 2: number of the isolate) showed a large zone of pigmentation $(38 \pm 0.5 \mathrm{~mm})$ after $24 \mathrm{~h}$ as compared to the other five isolates (supplementary Table 1), and hence, it was selected for further studies. The isolate DDB2 was gram-negative, motile, rod-shaped bacteria that produced melanin under alkaline conditions. The potential isolate DDB2 was identified by $16 \mathrm{~S}$ rRNA gene sequencing, and the phylogenetic tree (Fig. 1) was constructed using the neighborjoining method that showed a homology of $99 \%$ with Pseudomonas otitidis. The GenBank accession ID of $P$. otitidis DDB2 is MG585039. Figure 2 shows the extracellular dark brown pigment production by the isolate DDB2 on TGB agar. Carbohydrate utilization test showed that only glucose was utilized by the isolate

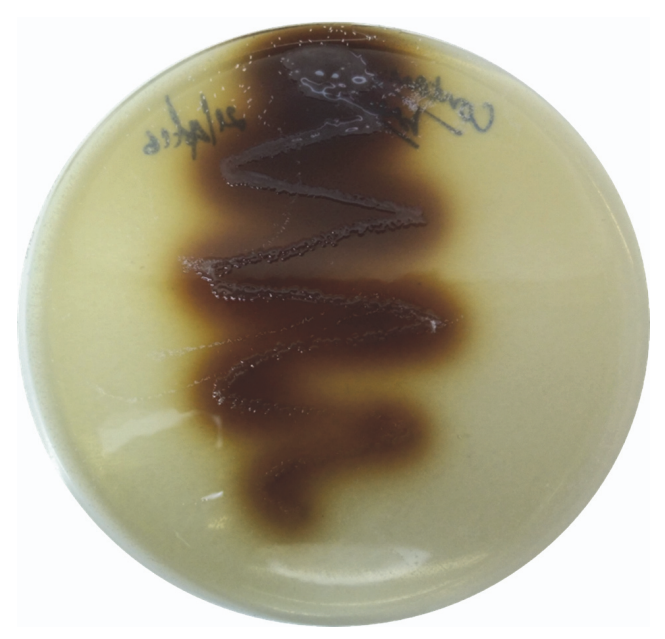

Fig. 2. Extracellular melanin produced by $P$. otitidis DDB2 on the TGB agar plate

DDB2, while other carbohydrate sources were not metabolized and no turbidity was observed (supplementary Table 2). The growth of DDB2 on M9 medium with glucose was compared with its growth in nutrient broth; the OD values of $0.24 \pm 0.02$ and $0.64 \pm 0.02$ were achieved in the former and latter media, indicating that the isolate poorly fermented sugars but actively utilized complex ingredients of nutrient broth.

\section{Optimization of melanin production}

The influence of physicochemical parameters such as $\mathrm{pH}$, temperature, L-tyrosine concentration, metal salts, 
Table 2. Solubility of melanin in different solvents tested

\begin{tabular}{c|c|c}
\hline $\begin{array}{c}\text { Series } \\
\text { number }\end{array}$ & Solvent & $\begin{array}{c}\text { Solubility } \\
\text { of melanin }\end{array}$ \\
\hline 1 & Water $(\mathrm{pH} \mathrm{7.0)}$ & insoluble \\
\hline 2 & $1 \mathrm{~N} \mathrm{HCl}$ & insoluble \\
\hline 3 & $1 \mathrm{~N} \mathrm{NaOH}$ & soluble \\
\hline 4 & $1 \mathrm{~N} \mathrm{NH}_{4} \mathrm{OH}$ & soluble \\
\hline 5 & DMSO & soluble \\
\hline 6 & DMF & soluble \\
\hline 7 & methanol & insoluble \\
\hline 8 & ethanol & insoluble \\
\hline 9 & n-butanol & insoluble \\
\hline 10 & ethyl acetate & insoluble \\
\hline 11 & acetone & insoluble \\
\hline 12 & n-hexane & insoluble \\
\hline 13 & diethyl ether & insoluble \\
\hline
\end{tabular}

and salinity on melanin production was assessed. The amount of melanin produced increased with the increase in $\mathrm{pH}$ from 6.0 to 8.0 , but gradually decreased from $\mathrm{pH}$ 9.0 to 10.0 (Fig. 3A). Melanin production exponentially increased from 30 to $40^{\circ} \mathrm{C}$, and a further increase in temperature to $45^{\circ} \mathrm{C}$ slightly increased the production of melanin by $0.13 \mathrm{~g} / \mathrm{l}$ (Fig. 3B). However, the growth of P. otitidis DDB2 was completely inhibited at $50^{\circ} \mathrm{C}$. The use of the precursor L-tyrosine resulted in the maximum production of melanin $(4.07 \pm 0.07 \mathrm{~g} / \mathrm{l})$ at the concentration of $1.5 \mathrm{~g} / \mathrm{l}$ (Fig. 3C). However, the absence of L-tyrosine resulted in no melanin formation $(0.0 \mathrm{~g} / \mathrm{l})$, and hence, this precursor was found to be mandatory for the synthesis of melanin by P.otitidis DDB2. Among the different nitrogen sources tested, $0.3 \%$ of beef extract or gelatin was found to exert a similar positive effect on the amount of melanin production $(4.6 \pm 0.10$ and $4.51 \pm 0.10 \mathrm{~g} / \mathrm{l}$, respectively). Therefore, either beef extract or gelatin at the concentration of $0.3 \%$ was included in the final medium for melanin production. Casein was found to produce the least amount of melanin $(1.56 \pm 0.10 \mathrm{~g} / \mathrm{l})$ than the other nitrogen sources tested (Fig. 3D). The results also showed that $0.01 \% \mathrm{CuCl}_{2}$ significantly affects melanin production by $P$. otitidis DDB2 (Fig. 3E). The metal salts $\mathrm{CoCl}_{2}$ and $\mathrm{CuSO}_{4}$ showed moderate increment in melanin production, whereas $\mathrm{MnCl}_{2}$ lowered melanin production by $2.54 \pm 0.12 \mathrm{~g} / \mathrm{l}$. Complete inhibition of me- lanin synthesis was observed with $\mathrm{MgCl}_{2}$. The salinity range of $0-2 \%$ neither enhanced nor reduced melanin production. Because the presence of $\mathrm{NaCl}$ did not greatly influence the amount of melanin production, it was excluded from the final optimized medium. The optimized conditions for the maximum production of melanin were found to be $\mathrm{pH} 8.0$, incubation temperature $45^{\circ} \mathrm{C}$, L-tyrosine $(1.5 \mathrm{~g} / \mathrm{l})$, beef extract $(0.3 \%)$, and $\mathrm{CuCl}_{2}$ $(0.01 \%)$. Melanin production by $P$. otitidis DDB2 increased from $3.81 \pm 0.1 \mathrm{~g} / 1$ to $5.3 \pm 0.1 \mathrm{~g} / 1$ under the optimized conditions.

\section{Characterization of melanin}

Purified melanin was soluble in alkaline solutions and in solvents such as DMSO and DMF, but it was insoluble in all the other solvents tested (Table 2). Melanin was precipitated by $\mathrm{FeCl}_{3}$ and turned colorless after reacting with $\mathrm{H}_{2} \mathrm{O}_{2}$. The requirement of L-tyrosine as the precursor, the dark brown color, and the solubility profile of the compound revealed that the pigment extracted from $P$. otitidis DDB2 belonged to the class eumelanin/DOPA melanin.

The UV-Vis absorption spectrum of melanin showed the maximum absorbance between the wavelength range of 215-350 nm (Fig. 4). The chromatogram developed on the TLC plate showed a broad spot with the retention factor (Rf) of 0.48 . HPLC with $0.1 \%$ formic acid and methanol (in the ratio of $85: 15$ ratio) as the mobile phase showed three major peaks with retention time of 1.620 , 2.273 , and 2.693 min corresponding to $10.01 \%, 63.20 \%$, and $17.42 \%$ of melanin constituents, respectively (supplementary Fig. 1).

\section{Antibacterial activity of melanin}

Purified melanin was assessed for its antibacterial activity (Fig. 5). A maximum diameter of $13 \pm 2 \mathrm{~mm}$ for the zone of inhibition was observed for $300 \mu \mathrm{g}$ of melanin against $S$. entericus, whereas the diameter of $12 \pm 2 \mathrm{~mm}$ was observed for the zones of inhibition with $300 \mu \mathrm{g}$ of melanin against $E$. coli, $P$. aeruginosa, and $B$. subtilis (supplementary Table 3 ). The zones of inhibition were $12 \pm 1 \mathrm{~mm}$ in diameter for $250 \mu \mathrm{g}$ of melanin against $E$. coli, $P$. aeruginosa, and $S$. entericus. For all the other tested concentrations of melanin, the diameter of zones of inhibition was $11 \pm 1 \mathrm{~mm}$ against the tested pathogenic bacteria. The zones of inhibition for the positive control streptomycin were $22 \pm 1,24 \pm 2,19 \pm 2$, 

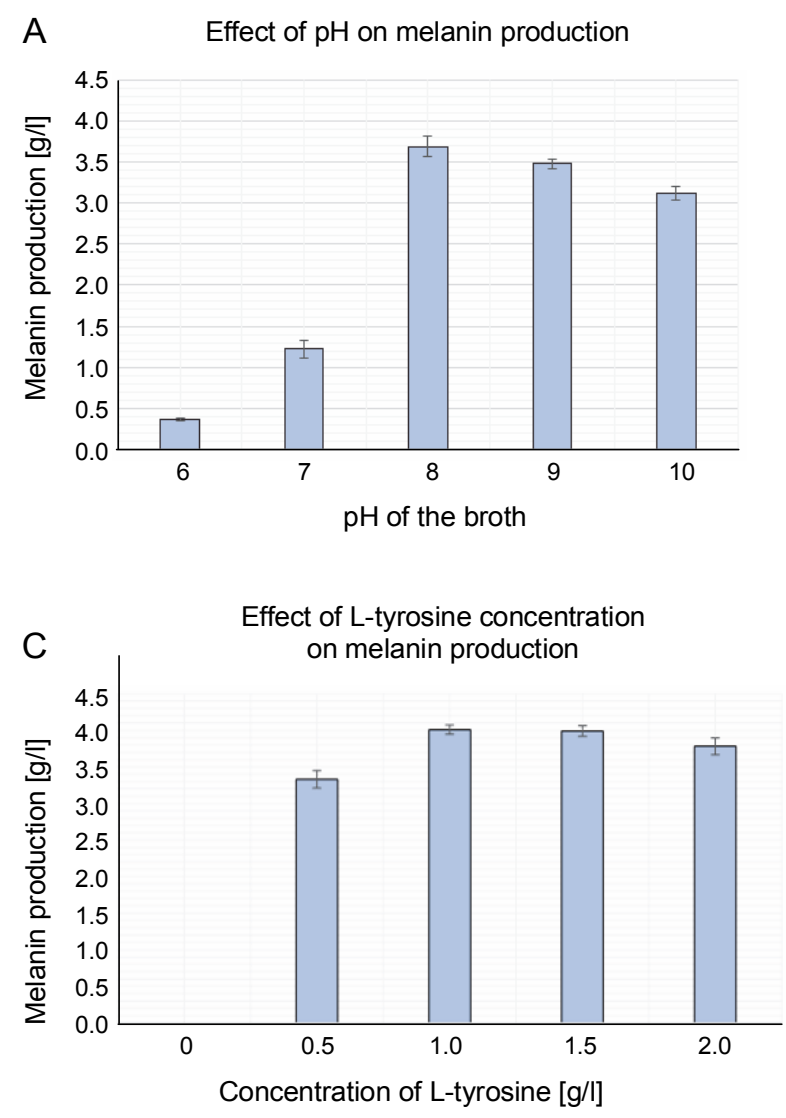

D Effect of metal salts on melanin production

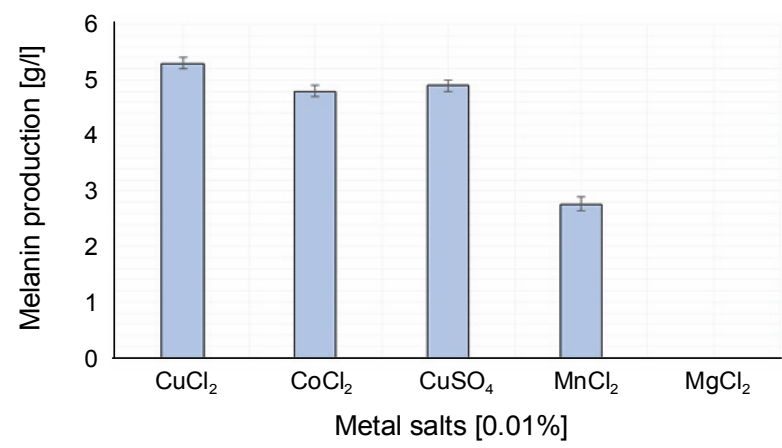

and $18 \pm 1 \mathrm{~mm}$ against $E$. coli, $P$. aeruginosa, $S$. entericus, and $B$. subtilis, respectively, whereas the negative control DMSO did not show any inhibition zone.

\section{Comparative analysis of tyrosinase gene sequences}

Tyrosinase gene sequences of selected organisms retrieved from NCBI were aligned, and a phylogenetic relationship was established to determine the relatedness of the tyrosinase gene of $H$. sapiens with that of other organisms such as Pseudomonas sp., Streptomyces sp., several fungal species, $S$. officinalis, Vibrio

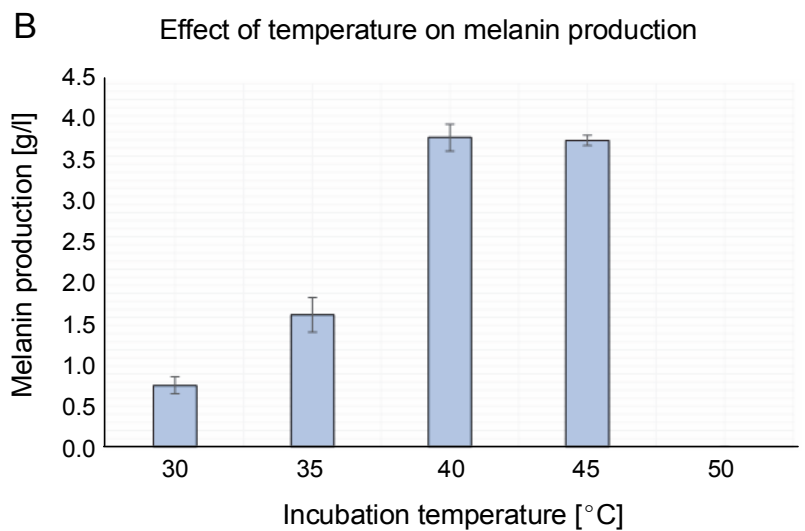

D Effect of nitrogen sources on melanin production

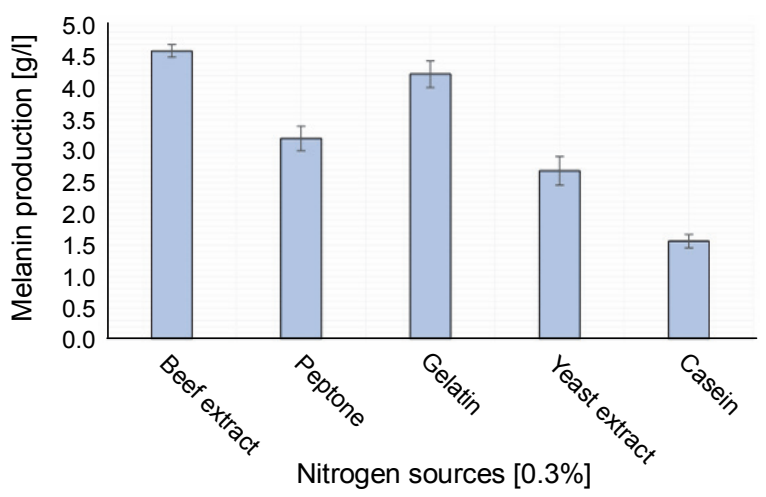

Fig. 3. Optimization of process variables for melanin production by $P$. otitidis DDB2. A) effect of $\mathrm{pH}$ on melanin production; $\mathrm{B}$ ) effect of temperature on melanin production; C) effect of L-tyrosine concentration on melanin production; D) effect of nitrogen sources on melanin production; E) effect of metal salts on melanin production

sp., and tyrosinase-related protein 1 (TYRP1) of $H$. sapiens. The resultant phylogenetic tree (Fig. 6) showed that the difference in the tyrosinase gene sequences increased in clockwise direction with respect to $H$. sapiens. The distance matrix was generated by Geneious software for the tyrosinase gene, protein, and CCD sequences, which showed the distances between the sequences of organisms based on percentage identity (\%) of the nitrogen bases or amino acid residues (supplementary Table 4). 


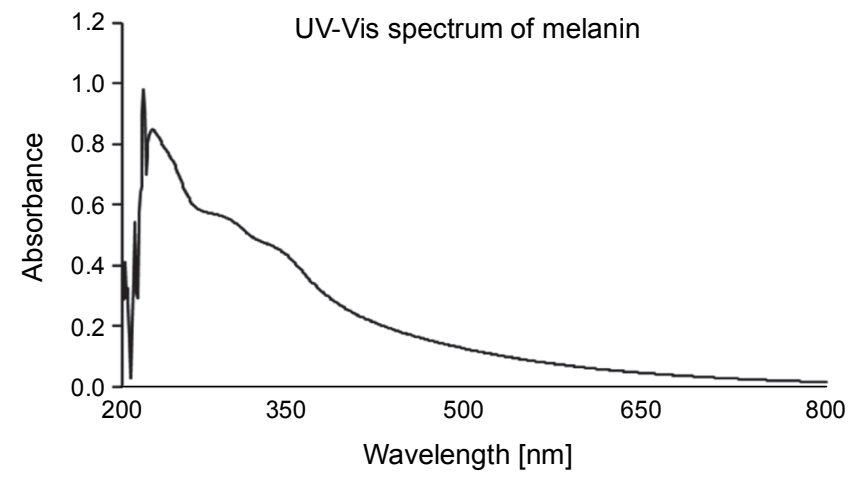

Fig. 4. UV-Vis absorption spectrum of melanin derived from $P$. otitidis DDB2

\section{Comparative analysis of tyrosinase gene sequences of selected organisms with the tyrosinase gene sequence of $\mathrm{H}$. sapiens (NM 000372.5)}

Among all the selected organisms, the TYRP1 gene sequence of $H$. sapiens was found to be more related to the tyrosinase gene sequence of selected $H$. sapiens with $34.7 \%$ identical bases, whereas $S$. officinalis showed $21.9 \%$ and $V$. nigripulchritudo showed $14.6 \%$ identity. Among the selected fungi, T. marneffei ATCC 18224 showed the highest similarity (20.5\%) and G. morbida showed the lowest similarity (1.8\%) with $H$. sapiens. Among the selected species of Streptomyces, S. subrutilus and $S$. fungicidicus showed the maximum (12.4\%) and the least $(0.6 \%)$ percentage of identity, respectively. On the other hand, $P$. proteolytica and $P$. frederiks bergensis showed the highest (14.6\%) and the least (6\%) similarity with the tyrosinase gene sequence of $H$. sapiens, respectively.

Comparative analysis of tyrosinase protein sequences of selected organisms with the tyrosinase protein sequence of $H_{\text {. sapiens (NP_o00363.1) }}$

The TYRP1 sequence of $H$. sapiens showed 39.3\% identity, while those of $S$. officinalis and $V$. nigripulchritudo showed $12.3 \%$ and $11.9 \%$ similarity, respectively, with the tyrosinase protein sequence of $H$. sapiens. $A$. niger showed the highest (12.4\%) and $G$. morbida showed the lowest (7.9\%) identical amino acid residues among the selected fungi. $S$. azureus showed the maximum (22.9\%) and S. fungicidicus showed the least (3\%) percentage of identity, whereas among the selected species of Pseudomonas, $P$. fluorescens exhibited the highest (20.8\%) and $P$. gingeri showed the least (10.5\%) percentage of identity with the tyrosinase protein sequence of $H$. sapiens.
A

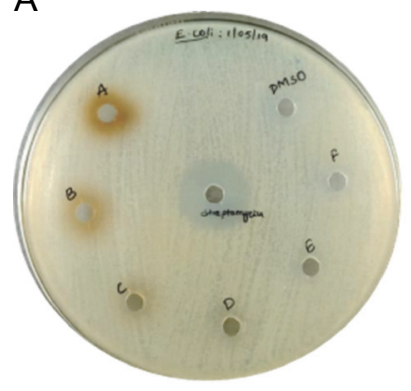

C

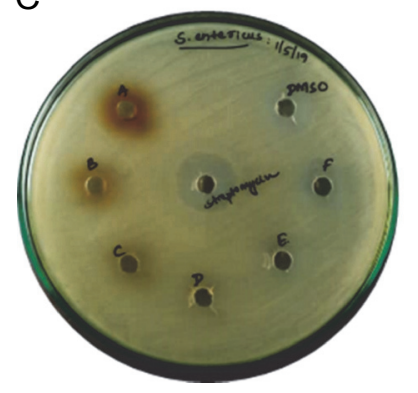

B

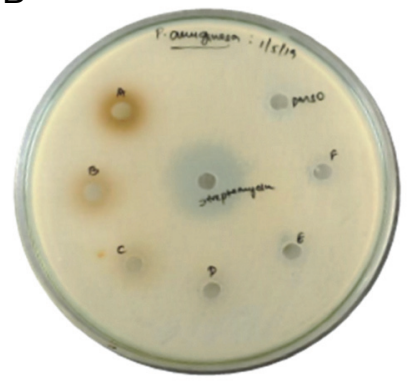

D

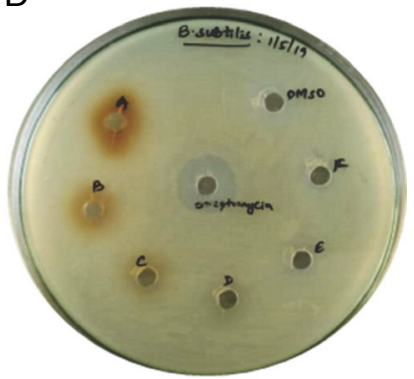

Fig. 5. Agar well assay for the antimicrobial activity of melanin (Plate A - E. coli; Plate B - P. aeruginosa; Plate C - S. entericus; Plate D - B. subtilis)

\section{Comparative analysis of $C C D$} of tyrosinase protein sequences

The CCD of the tyrosinase protein sequences of most selected organisms showed $100 \%$ identity with the CCD of $H$. sapiens (supplementary Table 3 ), and all these organisms exhibited common conserved residues at the positions 164-220 (PDQFRQNIPVLKNEVDRYLTNPSF VRFQADLDRGAHGAIHVFVGGQNSSSPLPANS). The CCD of $P$. putida, $P$. chlororaphis, and $P$. gingeri showed $56.35,61.4$, and $74.2 \%$ identity, respectively, with that of $H$. sapiens, respectively. However, certain species of Streptomyces such as $S$. zinciresistens, $S$. pluripotens, $S$. olivochromogenes, $S$. virginae, $S$. canus, $S$. avermitilis, $S$. cyaneus, $S$. violaceoruber, $S$. katrae, $S$. fodineus, $S$. fungicidicus, and $S$. wuyuanensis and the fungal species $G$. morbida exhibited no identity with the CCD of human tyrosinase protein sequence. Although these species did not harbor a CCD similar to that of $H$. sapiens, they were found to possess a conserved amino acid sequence (from position 31 to 73, AAMYEISELAVGDK RGYSALARAHGYDQDLCHDLDWAFLTWHR) that is commonly observed in other microorganisms selected in the present study. The multiple alignment of CCDs of the selected organisms is shown in Figure 7. 


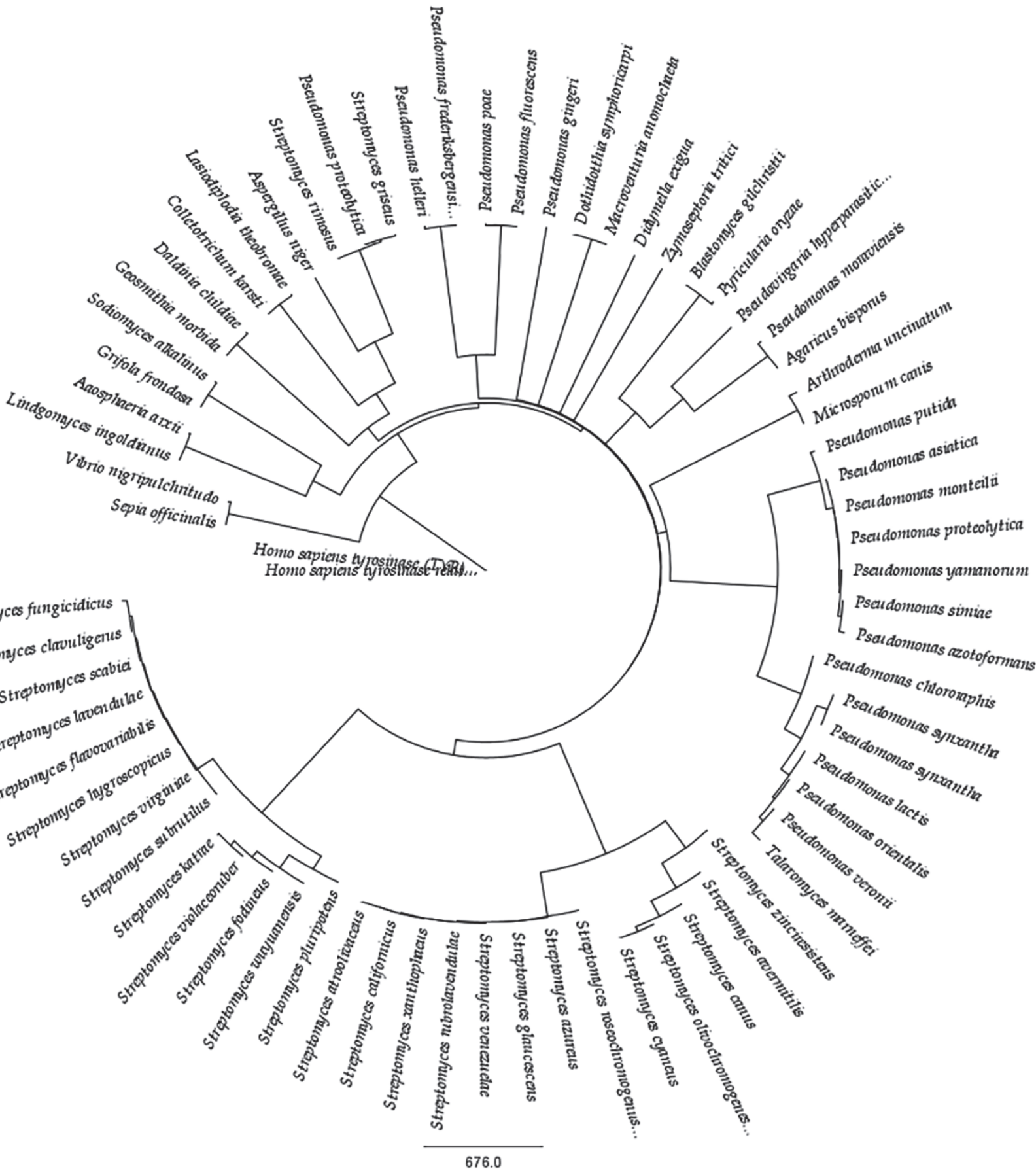

Fig. 6. Phylogenetic tree showing relatedness of tyrosinase gene sequences from selected organisms

\section{Discussion}

From preliminary screening, a single isolate named DDB2 showing the largest zone of extracellular melanin production was selected as the potential isolate and used for further studies. The other five isolates that produced maximum zones of pigmentation after $72 \mathrm{~h}$ of incubation were excluded from the study because the time of pig- ment production was too long and the diameter of the zone of pigmentation was smaller than that for the isolate DDB2. The isolate $P$. otitidis DDB2 could not utilize the carbohydrates provided, except glucose. However, it was observed that DDB2 poorly utilized glucose, but exhibited a rich growth on nutrient agar medium, which indicated the organism's preference for complex nu- 


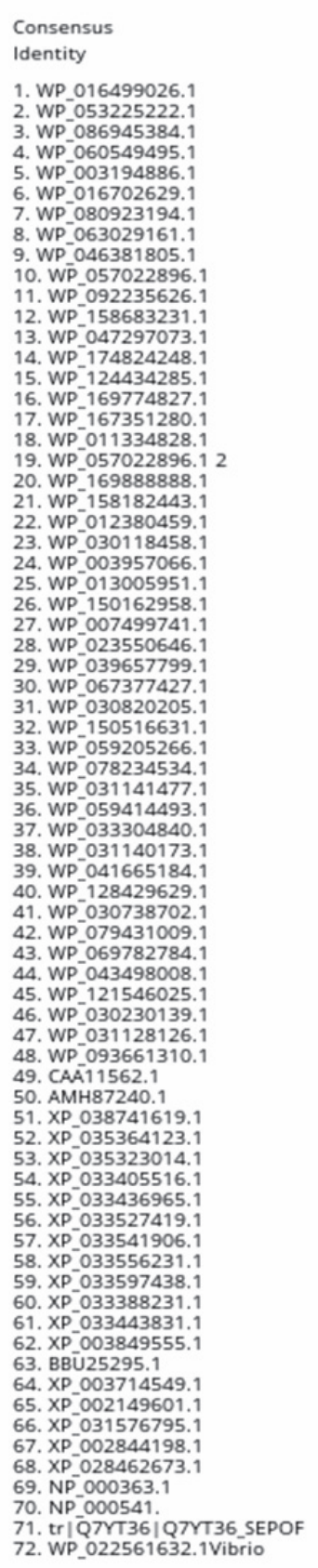

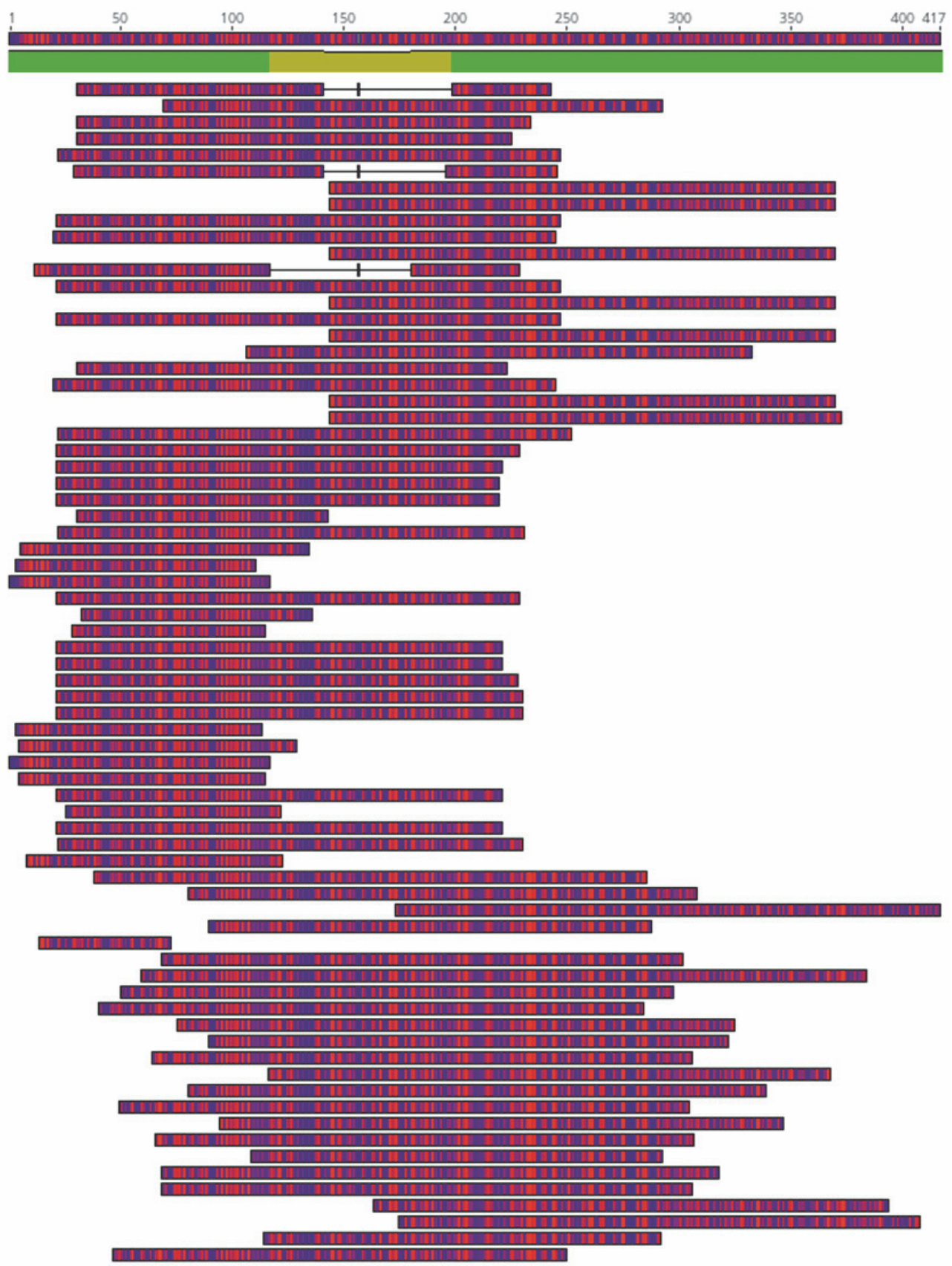

Fig. 7. Aligned sequences of $\mathrm{CCD}$ of the tyrosinase protein from selected organisms

trients. The genus $P$ seudomonas prefers organic acids or amino acids over sugars as their carbon source (Daniels et al., 2010). $P$. otitidis DDB2 produced the maximum amount of melanin at an alkaline $\mathrm{pH}$ of $8.0(3.81 \pm 0.1 \mathrm{~g} / \mathrm{l})$ and a high temperature of $45^{\circ} \mathrm{C}(3.85 \pm 0.1 \mathrm{~g} / \mathrm{l})$. A similar requirement of alkaline $\mathrm{pH}(9.0)$ and temperature $45^{\circ} \mathrm{C}$ was observed for melanin production by Streptomyces sp. isolated from limestone quarries in Deccan traps (Quadri and Agsar, 2012). Previous studies have shown that microorganisms produce melanin under certain stress conditions such as poor nutrient supply, high temperature, or high osmotic pressure (Fogarty and Tobin, 1996; Tran-Ly et al., 2020). This may be the reason for the requirement of high temperature $\left(40 \pm 5^{\circ} \mathrm{C}\right)$ and high $\mathrm{pH} \geq 8.0$ for melanin production by the isolated $P$ seudomonas sp. in this study. The isolate $P$. otitidis DDB2 showed an obligatory requirement of the precursor L-tyrosine for melanin production. Previous reports show that Pseudomonas stutzeri HMGM-7 and Pseudomonas sp. WH001 55 also produced melanin in the pre- 
sence of L-tyrosine (Ganesh Kumar et al., 2013; Kiran et al., 2017). The prerequisite of L-tyrosine as the precursor is a common feature of eumelanin-synthesizing organisms (Tran-Ly et al., 2020). It was observed that beef extract was consumed more readily by the isolate and facilitated the high titer of melanin production $(4.6 \pm 0.1 \mathrm{~g} / \mathrm{l})$. A similar requirement of beef extract for enhanced melanin production $(5.29 \mathrm{~g} / \mathrm{l})$ by $S$. lusitanus DMZ3 was reported previously (Madhusudhan et al., 2014). Copper ions are important cofactors for the functioning of melanogenic enzymes such as tyrosinases and laccases (Sendovski et al., 2011; Yang et al., 2017). The addition of $\mathrm{CuCl}_{2}(0.01 \%)$ significantly increased the yield of melanin produced by the isolate $P$. otitidis DDB2 by $0.7 \mathrm{~g} / \mathrm{l}$. Enhanced production of melanin can also be achieved with other metals by inducing stress in microorganisms (Gowri and Srivastava, 1996). For instance, Auricularia auricula and Streptomyces glaucescens NEAE-H require magnesium and iron salts, respectively, for enhanced melanin production (Sun et al., 2016; El Naggar and El-Ewasy, 2017). Surwase et al. (2013) reported improved production of melanin $(6.8 \mathrm{~g} / \mathrm{l})$ by Brevundimonas sp. SGJ in the presence of L-tyrosine, tryptone, and $\mathrm{CuSO}_{4}$ after $54 \mathrm{~h}$ of incubation. In another study, the fungus Armillaria cepistipes produced the maximum amount of melanin $(27.98 \mathrm{~g} / \mathrm{l})$ after 161 days of incubation and in the presence of carbon-nitrogen sources such as glucose and yeast extract along with metal salts of copper, iron, and magnesium (Ribera et al., 2019). The present study showed the maximum production of melanin $(5.3 \pm 0.1 \mathrm{~g} / \mathrm{l})$ by $P$. otitidis DDB2 with minimum medium ingredients and a short period of incubation $(24 \mathrm{~h})$ as compared to those reported earlier.

The extracted and purified melanin from $P$. otitidis DDB2 was found to be insoluble in most of the solvents tested, and this property of poor solubility is peculiar to melanin. The precipitation of melanin after reacting with $\mathrm{FeCl}_{3}$ indicated the presence of polyphenolic groups (Pralea et al., 2019). The melanin obtained in this study was degraded by alkaline $\mathrm{H}_{2} \mathrm{O}_{2}$, which was indicated by its bleaching. Purified melanin showed higher absorbance in UV range (Fig. 4), which decreased gradually with further increase in wavelength toward the visible region; this attribute is unique to melanin (Meredith and Sarna, 2006; Al Khatib et al., 2018). We characterized melanin extracted from $P$. otitidis DDB2 by other methods such as thermal stability, elemental analysis,
FT-IR, and NMR as reported earlier (Seelam et al., 2021). Our previous studies on the characterization of melanin revealed its basic constituent as 5,6-dihydroxy indole, 2-carboxylic acid (Seelam et al., 2021). The three peaks observed in chromatogram of HPLC may be attributed to the oligomers of melanin with varying number of monomeric units. The broad spot developed on chromatogram of TLC and multiple peaks observed in HPLC show the heterogenous nature of melanin under study. It is a common feature of natural melanin to exhibit the property of heterogeneity and insolubility due to the complex arrangement of its molecular structure (Pralea et al., 2019).

In the present study, no antibacterial activity of melanin was detected against the tested pathogens $E$. coli, $P$. aeruginosa, $S$. entericus, and $B$. subtilis. The diameters of inhibition were very low as compared to the positive control streptomycin. Antibacterial activity of melanin in the present study showed contrasting results to earlier reports. For instance, melanin obtained from the fungus Schizophyllum communae exhibited antibacterial activity against $E$. coli, Klebsiella pneumoniae, Proteus sp., and Pseudomonas fluorescens at the concentration range of 50-200 $\mu \mathrm{g}$ (Arun et al., 2015). In another study, melanin (30 $\mu \mathrm{g} / \mathrm{disc})$ from Streptomyces sp. showed antibacterial activity against $P$. aeruginosa and Vibrio parahaemolyticus (Sivaperumal et al., 2014). Similarly, purified melanin from Actinoalloteichu s sp. MA32 exhibited antibacterial activity against $B$. subtilis and $E$. coli at the concentration of 30 and $50 \mu \mathrm{g} /$ disc (Manivasagan et al., 2013). Lopusiewicz (2018) reported the antibacterial activity of purified melanin extracted from Armillaria sp. against B. cereus, B. subtilis, Enterococcus faecalis, and $P$. aeruginosa. However, no activity was observed against $B$. anthrophaeus, E. coli, Micrococcus luteus, $P$. putida, and $S$. aureus. These incongruities in the antibacterial activity of melanin may result from the differences in the composition and molecular structure of melanin (Correa et al., 2017).

Recent advances in gene sequencing approaches and the development of computational biology tools have enabled the researchers to study the diversity of genes and understand the evolutionary relationship among different species. In the present study, an attempt was made to comparatively analyze tyrosinase gene and protein sequences of selected organisms with $H$. sapiens. The rationale underlying the selection of organisms for 
this study was that the potential organism in the present study was identified as Pseudomonas sp., and hence, different species of the same genus were selected for comparative analysis. Several studies have shown that the other common microbial sources for tyrosinase or melanin are Streptomyces (Madhusudhan et al., 2014; El Naggar and El-Ewasy 2017), Vibrio (Vijayan et al., 2017; Kurian and Bhat, 2018), or fungal species (Mattoon et al., 2021), and hence, they were also included in the study. S. officinalis was selected because this is the only source of commercially available natural melanin (Guo et al., 2014). The TYRP1 sequences of $H$. sapiens involved in melanin synthesis, maintenance of melanosome structure, and stabilization of tyrosinase were also selected as parameters to determine how likely TYRP1 and tyrosinase sequences of $H$. sapiens are related despite their location on different chromosomes (Zheng et al., 2010). However, the sequences of $H$. sapiens used as the out group were arbitrarily selected.

The distance matrix of the aligned sequences showed that the TYRP1 gene of $H$. sapiens (34.7\%) is closer to tyrosinase of $H$. sapiens, followed by $S$. officinalis $(21.9 \%)$ and T. marneffei ATCC18224 (20.5\%). The protein sequences of TYRP1 of $H$. sapiens showed $39.3 \%$ identity, followed by $S$. azureus with $22.9 \%$ identity and $S$. clavuligerus with $22.7 \%$ identity with the tyrosinase protein of $H$. sapiens. The central domains of most of the species in the present study showed $100 \%$ identity with that of $\mathrm{CCD}$ of $H$. sapiens. Although a large variation was observed in the tyrosinase gene sequences of selected organisms, a $100 \%$ similarity in their central domains strongly implicate that the functional domains of the tyrosinase protein are highly conserved irrespective of their taxonomic positions. In silico studies provided insights on the variability of sequences and applicability of melanin to closely related species. Researchers from China have performed a similar analysis of TYRP1 among different species and showed that there were two evolutionarily conserved noncoding regions present upstream to the initiation sites of translation. They also reported the amino acids mutated within the species and the amino acids highly conserved among the species (Zheng et al., 2010). Therefore, the use of bioinformatics tools in scientific research aid in better understanding of evolutionary changes in a particular gene sequence and its directionality.

\section{Conclusions}

Melanin has recently gained significance for application in green technology and material science as an additive that can substantially enhance the performance of other materials. In light of its increasing demand, in the present study, we report the screening, isolation, and production of melanin by a potential strain of $P$. otitidis DDB2. The isolated melanin belonged to the eumelanin class with the maximum absorption range in the ultraviolet region, and hence, it could be used as a natural pigment in sunscreen products. Furthermore, in silico studies of melanogenic gene sequences provided us insights into the applicability of melanin to humans due to the evolutionary relatedness between the tyrosinase genes of humans and the selected Pseudomonas sp.

\section{Acknowledgments}

SSD is grateful to Council for Scientific and Industrial Research (CSIR), New Delhi, India, for the award of Senior Research Fellowship. The authors also thank Dr. Syed Dastager, NCIM Resource Center, CSIR-National Chemical Laboratory, Pune, India, and Dr. H. M. Sampath Kumar, CSIR-Indian Institute of Chemical Technology, Hyderabad, India, for their technical support.

\section{References}

Ahn S.Y., Choi M., Jeong D.W., Park S., Park H., Jang K.S., Choi K.Y. (2019) Synthesis and chemical composition analysis of protocatechualdehyde-based novel melanin dye by $15 T$ FT-ICR: high dyeing performance on soft contact lens. Dyes Pigments 160: 546-554.

Al Khatib M., Harir M., Costa J., Baratto M.C., et al. (2018) Spectroscopic characterization of natural melanin from a Streptomyces cyaneofuscatus strain and comparison with melanin enzymatically synthesized by tyrosinase and laccase. Molecules 23(8): 1916.

Ali Amal M., Keera Abeer A., Helmy Samia M., Abd El-Nasser Nadia H., Ahmed K.A., El-Hennawi H.M. (2011) Selection of pigment (melanin) production in Streptomyces and their application in printing and dyeing of wool fabrics. Res. J. Chem. Sci. 1(5): 22-28.

Arun G., Eyini M., Gunasekaran (2015) Characterization and biological activities of extracellular melanin produced by Schizophyllum commune (Fries). IJEB. 53: 380-387.

Banerjee A., Supakar S., Banerjee R. (2014) Melanin from the nitrogen-fixing bacterium Azotobacter chroococcum: a spectroscopic characterization. PLoS One. 9(1): e84574.

Bartholomew J.W., Mittwer T. (1952) The Gram stain. Bacteriol. Rev. 16(1): 1-29.

Battistella C., Naneki Mc C.C., Gnanasekaran K., Zhou X., Caponetti V., Montalti M., Nathan Gianneschi C. (2020) 
Mimicking natural human hair pigmentation with synthetic melanin. ACS Cent. Sci. 6(7): 1179-1188.

Cordero R.J.B, Casadevall. (2017) A functions of fungal melanin beyond virulence. Fungal Biol. Rev. 31: 99-112.

Correa N., Covarrubias C., Rodas P.I., Hermosilla G., Olate V.R., Valdés C., Meyer W., Magne F., Tapia C.V. (2017) Differential antifungal activity of human and Cryptococcal melanins with structural discrepancies. Front. Microb. 8: 1-13. https://doi.org/10.3389/fmicb.2017.01292

D’Ischia M., Pezzella A., Napolitano A., et al. (2015) Melanins and melanogenesis: from pigment cells to human health and technological applications. Pigment Cell Melanoma Res. 28: 520-544.

Daniels C., Godoy P., Duque E., Molina-Henares M.A., De La Torre J., Del Arco J.M., Herrera C., Segura A., Guazzaroni M.E., Ferrer M. et al. (2010) Global regulation of food supply by Pseudomonas putida DOT-T1E. J. Bacteriol. 192: 2169-2181

Drewnowska J.M., Zambrzycka M., Kalska-Szostko B., Fiedoruk K., Swiecicka I. (2015) Melanin-like pigment synthesis by soil bacillus weihenstephanensis isolates from northeastern Poland. PLoS One 10(4): e0125428.

Eisenman H.C., Mues M., Weber S.E., Frases S., Chaskes S., Gerfen G., et al. (2007) Cryptococcus neoformans laccase catalyses melanin synthesis from both $D$ - and L-DOPA. Microbiology 153: 3954-3962.

El-Naggar N.E.A, El-Ewasy S.M. (2017) Bioproduction, characterization, anticancer and antioxidant activities of extracellular melanin pigment produced by newly isolated microbial cell factories Streptomyces glaucescens NEAEH. Sci. Rep. 7: 42129.

Esposito R., D’Aniello S., Squarzoni P., Pezzotti M.R., Ristoratore F., Spagnuolo A. (2012) New insights into the evolution of metazoan tyrosinase gene Family. PLoS One 7(4): e35731.

Fava F., Gioia D.D., Marchetti L. (1993) Characterization of a pigment produced by Pseudomonas florescens during 3-chlorobenzoate co-metabolism. Chemosphere 27(5): 825-835.

Fogarty R.V., Tobin J.M. (1996) Fungal melanins and their interactions with metals. Enzyme Microbiol. Technol. 19: 311-317.

Ganesh Kumar C., Sahu N., Narender Reddy G., Prasad R.B.N., Nagesh N., Kamal A. (2013) Production of melanin pigment from Pseudomonas stutzeri isolated from red seaweed Hypnea musciformis. Lett. Appl. Microbiol. 57: 295-302.

Gibson L.F., George A.M. (1998) Melanin and novel melanin precursors from Aeromonas media. FEMS Microbiol. Lett. 169(2): 261-268.

Gowri P.M., Srivastava S. (1996) Encapsulation as a response of Azospirillum brasilense sp7 to zinc stress. World J. Microbiol. Biotechnol. 12: 319-322. https://doi.org/ 10.1007/BF00340207

Guo J., Rao Z., Yang T., Man Z., Xu M., Zhang X. (2014) Highlevel production of melanin by a novel isolate of Streptomyces kathirae. FEMS Microbiol. Lett. 357: 85-91.
Jalmi P., Bodke P., Wahidullah S., Raghukumar S. (2012) The fungus Gliocephalotrichum simplex as a source of abundant, extracellular melanin for biotechnological applications. World J. Microbiol. Biotechnol. 28: 505-512. https:// doi.org/10.1007/s11274-011-0841-0

Kamal U.Z., Ayesha Ali S., Sharique Ali A., Ishrat N. (2014) Microbial tyrosinases: promising enzymes for pharmaceutical, food bioprocessing, and environmental industry. Biochem. Res. Int. 014: 854687.

Kiran G.S., Dhasayan A., Lipton A.N., Selvin J., Arasu M.V., Al-Dhabi N.A. (2014) Melanin-templated rapid synthesis of silver nanostructures. J. Nanobiotechnol. 12: 1.

Kiran G.S., Jackson S.A., Priyadharsini S., Alan Dobson D.W., Selvin J. (2017) Synthesis of Nm-PHB (nanomelanin-polyhydroxy butyrate) nanocomposite film and its protective effect against biofilm-forming multi drug resistant Staphylococcus aureus. Sci. Rep. 7: 9167.

Kotob S.I., Coon S.L., Quintero E.J., Weiner R.M. (1995) Homogentisic acid is the primary precursor of melanin synthesis in Vibrio cholerae, a Hyphomonas strain, and Schwenella colwelliana. Appl. Environ. Microbiol. 61: 1620-1622.

Kurian N.K., Bhat S.G. (2018) Food, cosmetic and biological applications of characterized DOPA-melanin from Vibrio alginolyticus strain BTKKS3. Appl. Biol. Chem. 61(2): 163-171.

Langfelder K., Jahn B., Gehringer H., Schmidt A., Wanner G., and Brakhage A.A. (1998) Identification of a polyketide synthase gene ( $p k s P$ ) of Aspergillus fumigatus involved in conidial pigment biosynthesis and virulence. Med. Microbiol. Immunol. 187: 79-89.

Lopusiewicz L. (2018) The isolation, purification and analysis of the melanin pigment extracted from Armillaria mellea rhizomorphs. World Sci. News 100: 135-153.

Madhusudhan D.N., Bi Bi Zainab M., Syed D.G., Dayanand A. (2014) Production and cytotoxicity of extracellular insoluble and droplets of soluble melanin by Streptomyces lusitanus DMZ-3. J. Biomed. Biotechnol. 5: 306895.

Manivasagan P., Venkatesan J., Senthilkumar K., Sivakumar K., Kim S.K. (2013) Isolation and characterization of biologically active melanin from Actinoalloteichus sp. MA-32. Int. J. Biol. Macromol. 58: 263-274. https://doi.org/ 10.1016/j.ijbiomac.2013.04.041

Mattoon E.R., Cordero R.J.B., Casadevall A. (2021) Fungal melanins and applications in healthcare, bioremediation and industry. J. Fungi. 7: 488. https://doi.org/10.3390/ jof7060488

Meredith P., Sarna T. (2006) The physical and chemical properties of eumelanin. Pigment Cell Res. 19: 572-594.

Pralea I.E., Moldovan R.C., Petrache A.M. et al. (2019) From extraction to advanced analytical methods: the challenges of melanin analysis. Int. J. Mol. Sci. 20: 3943.

Quadri S.R., Agsar D. (2012) Detection of melanin producing thermo-alkaliphilic Streptomyces from limestone quarries of the Deccan traps. World J. Sci. Technol. 2(2): 08-12.

Raman N.M., Shah P.H., Mohan M., Ramasamy S. (2015) Improved production of melanin from Aspergillus fumigatus 
AFGRD105 by optimization of media factors. AMB Express 5: 72 .

Ribera J., Panzarasa G., Stobbe A., Osypova A., Rupper P., Klose D., Francis Schwarze W.M.R. (2019) Scalable biosynthesis of melanin by the basidiomycete Armillaria cepistipes. J. Agric. Food Chem. 67: 132-139.

Romero-Martinez R., Wheeler M., Guerrero-Plata A., Rico G., Torres-Guerrero H. (2000) Biosynthesis and functions of melanin in Sporothrix schenckii. Infect. Immun. 68: 3696-3703.

Schleheck D., Barraud N., Klebensberger J., Webb J.S., Mc. Dougald D., Rice S.A., Kjelleberg S. (2009) Pseudomonas aeruginosa PAO1 preferentially grows as aggregates in liquid batch cultures and disperses upon starvation. PLoS One 4(5): e5513. https://doi.org/10.1371/journal.pone. 0005513

Seelam S.D., Agsar D., Sampath Kumar H.M., Prakasham R.S., Sravanthi V., Mohan Reddy K., Umesh M.K., Rajitha C.H. (2021) Characterization and photoprotective potentiality of lime dwelling Pseudomonas mediated melanin as sunscreen agent against UV-b radiations. J. Photochem. Photobiol. B: Biol. 216: 112126.

Sendovski M., Kanteev M., Ben-Yosef V.S., Adir N., Fishman A. (2011) First structures of an active bacterial tyrosinase reveal copper plasticity. J. Mol. Biol. 405: 227-237. https:// doi.org/10.1016/j.jmb.2010.10.048

Serrano D.L., Solano F., Sanchez-Amat A., (2004) Identification of an operon involved in tyrosinase activity and melanin synthesis in Marinomonas mediterranea. Gene 342: 179-187.

Shanmuganathan K., Cho J.H., Iyer P., Baranowitz S., Ellison C.J. (2011) Thermooxidative stabilization of polymers using natural and synthetic melanins. Macromolecules 44: 9499-9507. https://doi.org/10.1021/ma202170n

Shivaveerakumar S., Madhusudhan D.N., Agsar D., Kontro M. (2013) Catalytic zone, a novel screening approach for the extracellular tyrosinase by Streptomyces vinaceusdrappus DSV5. J. Pure Appl. Microbiol. 7: 2799-2808.

Singh D., Kumar J., Kumar A. (2018) Isolation of pyomelanin from bacteria and evidences showing its synthesis by 4-hydroxyphenylpyruvate dioxygenase enzyme encoded by hppD gene. Intl. J. Biol. Macromol. 119: 864-873.

Sivaperumal P., Kamala K., Rajaram R. (2014) Bioactive DOPA melanin isolated and characterised from a marine actinobacterium Streptomyces sp. MVCS6 from Versova coast. Nat. Prod. Res. 29: 2117-2121.

Sun S., Zhang X., Chen W., Zhang L., Zhu H. (2016) Production of natural edible melanin by Auricularia auricula and its physicochemical properties. Food Chem. 196: 486-492.
Surwase S.N., Jadhav S.B., Phugare S.S., Jadhav J.P. (2013) Optimization of melanin production by Brevundimonas $s p$. SGJ using response surface methodology. 3 Biotech 3: 187-194.

Thorat M.N. and Dastager S.G. (2018) High yield production of cellulose by a Komagataeibacter rhaeticus PG2 strain isolated from pomegranate as a new host. RSC advances. 8: 29797-29805. https://doi.org/10.1039/C8RA05295F

Toledo A.V., Franco M.E.E, Yanil Lopez S.M., Troncozo M.I., Saparrat M.C.N., Balatti P.A. (2017) Melanins in fungi: types, localization and putative biological roles. Physiol. Mol. Plant Pathol. 9: 2-6.

Tran-Ly A.N., Reyes C., Francis Schwarze W.M.R., Ribera J. (2020) Microbial production of melanin and its various applications. World J. Microbiol. Biotechnol. 36: 170.

Vasanthabharathi V., Lakshminarayanan R., Jayalakshmi S. (2011) Melanin production from marine Streptomyces. Afr. J. Biotech. 10(54): 11224-11234.

Vijayan V., Jasmin C., Anas A., Parakkaparambil Kuttan S., Vinothkumar S., Perunninakulath Subrayan P., Nair S. (2017) Sponge-associated bacteria produce non-cytotoxic melanin which protects animal cells from photo-toxicity. Appl. Biochem. Biotechnol. 183(1): 396-411.

Wang R., Wang H., Zhou H., Wang Y., Yue J., Diao B., Kan B. (2011) Characters of homogentisate oxygenase gene mutation and high clonality of the natural pigment-producing Vibrio cholerae strains. BMC Microbiol. 11: 109.

Williams R.F. (1994) Melanin-based agents for image enhancement. U.S. Patent No. 5310539.

Woo P.C., Tam E.W., Chong K.T., Cai J.J., Tung E.T., Ngan A.H., Lau S.K., Yuen K.Y. (2010) High diversity of polyketide synthase genes and the melanin biosynthesis gene cluster in Penicillium marneffei. FEBS. J. 277:3750-3758.

Yang J., Li W., Bun Ng T., Dend X., Lin J., Ye X. (2017) Laccases: production, expression regulation, and applications in pharmaceutical biodegradation. Front. Microbiol. 8: 832. https://doi.org/10.3389/fmicb.2017.00832

Zerrad A., Anissi J., Ghanam J., Sendide K., El Hassouni M. (2014) Antioxidant and antimicrobial activities of melanin produced by a Pseudomonas balearica strain. J. Biotech. Lett. 5(1): 087-094.

Zheng H., Li X., Zhou R., Li L., Guo X., Kang J., Li D. (2010) Bioinformatics analysis of tyrosinase-related protein 1 gene (TYRP1) from different species. Front. Agric. China. 4(1): 109-115. 